$\mathrm{DE}$

M E D I C I N A

T R O P I C A L

$\mathrm{DE}$

SÃO PAULO

JOURNAL OF THE SÃO PAULO INSTITUTE OF TROPICAL MEDICINE

'Universidade de São Paulo, Faculdade de Medicina de Ribeirão Preto, Hemocentro de Ribeirão Preto, Ribeirão Preto, São Paulo, Brazil

${ }^{2}$ Instituto para Pesquisa do Câncer, Guarapuava, Paraná, Brazil

${ }^{3} \mathrm{AC}$ Camargo Cancer Center, Centro Internacional de Pesquisa, Facility de Sequenciamento Genômico, São Paulo, São Paulo, Brazil

Correspondence to: Svetoslav Nanev Slavov

Universidade de São Paulo, Faculdade de Medicina de Ribeirão Preto, Hemocentro de Ribeirão Preto, Rua Tentente Catão Roxo, 2501, CEP 14051-140, Ribeirão Preto, SP, Brazil

Tel: +55162101.9300

Fax: +55 162101.9309

E-mail:

svetoslav.slavov@hemocentro.fmrp.usp.br

Received: 26 August 2020

Accepted: 27 August 2020

\section{Viral metagenomics in blood donors with post-donation diseases and negative tests for dengue and Zika viruses RNA detection during a major outbreak of arboviruses in Sao Paulo State in 2016}

Ribeirao Preto, August 26 ${ }^{\text {th }}, 2020$
Dear Editor

Post-donation illness report (PDIR) is characterized by a voluntary call of the blood donor back to the blood collection institution due to the manifestation of clinical symptoms compatible with infectious disease long after the donation. Studies examining PDIR by viral metagenomics demonstrate that viral agents can be frequently involved in PDIR events and these include dengue viruses (DENV), parvovirus B19 (B19V) and influenza $A^{1,2}$. Apart from this, the great majority of PDIR etiology remains unknown. It is impossible to routinely test all blood donations for all infectious agents that can be transmitted by blood transfusion, but highly complex methods such as next-generation sequencing (NGS) can identify blood transmissible viral agents with importance for hemotherapy ${ }^{3}$, and these methods can be applied to investigate PDIR causes. PDIR can bring important data on viral infectious agents that are potential transfusion threats and direct public health policies for elaboration of strategies to prevent their transfusion transmission.

Given this, in our study we investigated, by NGS and viral metagenomics, 46 PDIR plasma samples divided according to the following symptoms: the majority of them comprised PDIR of diarrhea ( $n=16 / 46,34.8 \%)$, followed by PDIR characterized by fever ( $\mathrm{n}=13 / 46,28.3 \%$ ), respiratory symptoms (coryza, sore throat, flu-like symptoms), $n=12 / 46,26.1 \%$ and exanthema $(n=5 / 46,10.9 \%)$. All of the samples were obtained between January-April, 2016 during an extensive DENV/Zika virus (ZIKV) outbreak in the Northeast region of Sao Paulo State, Brazil, where the city of Ribeirao Preto is located, and samples that tested positive for DENV and/or ZIKV virus RNA were not included in the study due to the identification of the causing agent.

The samples were extracted in pools composed of 6-7 plasma samples using the High Pure Viral Nucleic Acids Large Volume kit (Roche, Basel, Switzerland) following the manufacturer's instructions. Before extraction, plasma samples were treated with Turbo DNAse (Thermo Fisher Scientific, Waltham MA, USA) for host DNA removal. Reverse transcription was performed using Superscript III First-Strand Synthesis System (Thermo Fisher Scientific,Waltham, MA, USA) and the obtained cDNA was amplified by the QuantiTect Whole Transcriptome Kit (QIAGEN, Hilden, Germany). DNA libraries were generated using Nextera DNA Flex Library Preparation Kit (Illumina, San Diego, CA, USA) following the manufacturer's instructions. Sequencing was carried out in Illumina NextSeq 500 sequencer using the NextSeq High Output Kit v 2.5, 300 cycles (Illumina, San Diego, CA, USA).

The raw sequence data obtained were submitted to quality control analysis using the FastaQC software (version 0.11.8, Babraham Institute, UK). Trimming was performed using a Trimmomatic (version 0.3.9, Max Planck Institute, Jülich, Germany) in order to select the best quality sequences. For the bioinformatic analysis, we used sequences with quality scores $>30$. Additional trimming to improve the overall quality was performed using the AfterQC software (version 0.9.7, Shenzhen Institutes of Advanced Technology, Shenzhen, China). The 
Kraken2 program (version 2.0.8, Johns Hopkins University, Baltimore, USA) was used to determine the taxonomic classification. "De novo" assembly was performed using SPAdes (version 3.13.0, St. Petersburg Academic University, St. Petersburg, Russia) to generate contigs and scaffolds. Finally, to the perform taxonomic classification based on protein identity we applied Blastx according to the Diamond software (version 0.9.29, University of Tübingen, Tübingen, Germany).

A

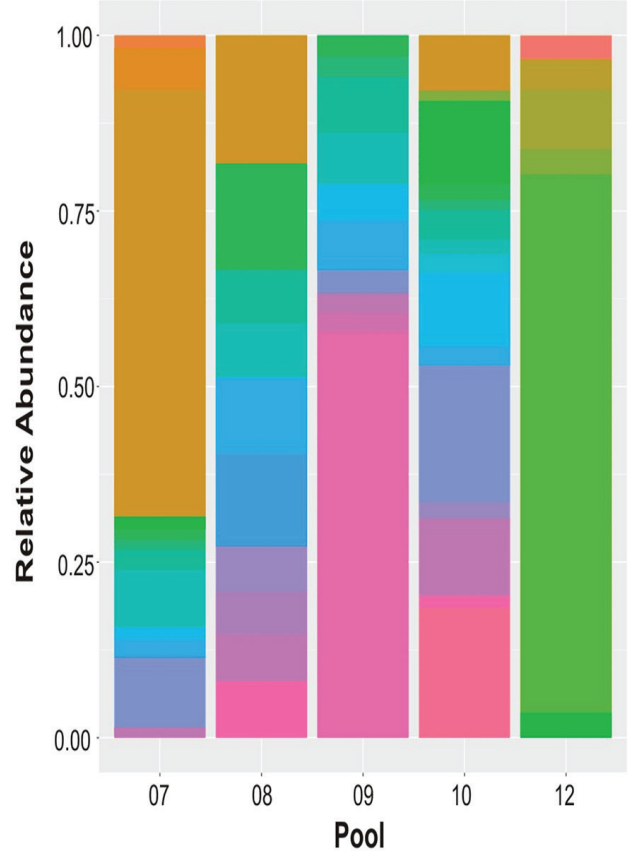

After performing the bioinformatics analysis, the viral diversity of the PDIR samples was verified. Among the non-pathogenic viruses found in the pools, we detected a strong presence of many genetically diverse representatives of the Anelloviridae family (Figure 1). Among the identified anelloviruses in our samples we can highlight torque teno viruses (TTV) - 3, 5, 8, 10-13, 15, 16, 19, 20, 24, 27-29 and torque teno mini virus (TTMV) 5 . The anellovirus abundance showed a similar distribution in all tested pools,

B

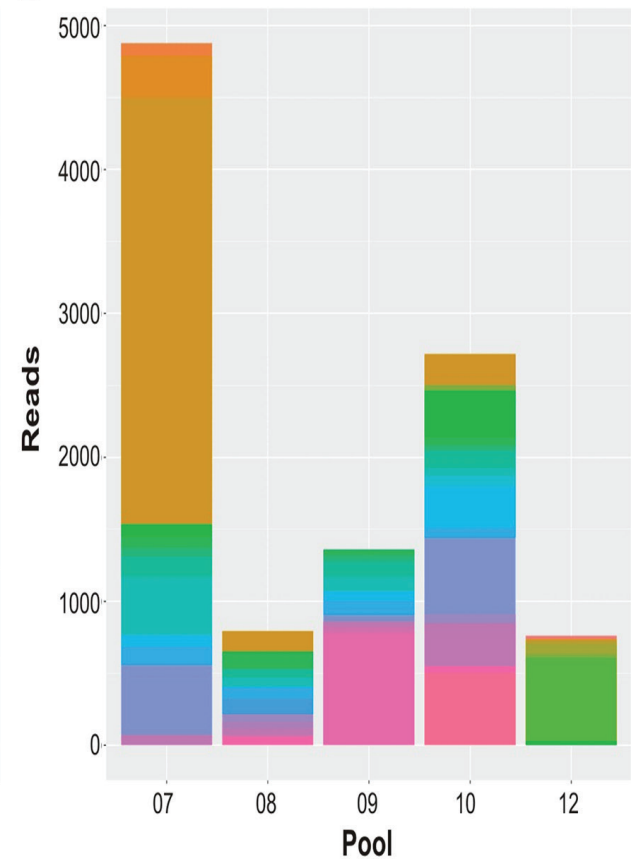

\begin{tabular}{|l|l|}
\hline Virus & \\
\hline Acanthamoeba polyphaga moumouvirus & Torque teno virus 15 \\
\hline Apocheima cinerarium nucleopolyhedrovirus & Torque teno virus 16 \\
\hline Bacillus virus G & Torque teno virus 19 \\
\hline Invertebrate iridescent virus 6 & Torque teno virus 20 \\
\hline Megavirus chiliensis & Torque teno virus 24 \\
\hline TTV-like mini virus & Torque teno virus 27 \\
\hline Orpheovirus IHUMI-LCC2 & Torque teno virus 28 \\
\hline Torque teno mini virus 5 & Torque teno virus 29 \\
\hline Torque teno virus & Torque teno virus 3 \\
\hline Torque teno virus 10 & Torque teno virus 5 \\
\hline Torque teno virus 11 & Torque teno virus 8 \\
\hline Torque teno virus 12 & Pseudomonas virus TL \\
\hline Torque teno virus 13 &
\end{tabular}

Figure 1 - Bar plot illustrating the relative and absolute abundance of viruses found in the tested pools of plasma samples. In the $\mathrm{X}$-axis is expressed the number of the tested pools: A) Relative abundance of viruses found in the analyzed pools; B) Absolute abundance in relation to the number of viral reads found in the tested pools. The high abundance of iridescent arthropod viruses can be observed in pool number 07 , to which a passive transmission of the circulating mosquito vectors during the tested arboviral outbreak can be attributed. 
i.e., similar anellovirus species were found in all tested samples and their sequence coverage was also similar.

During the metagenomic analysis, we also observed a high number of reads belonging to Pseudomonas spp phages. The phage diversity may be an indirect indicator of the pronounced bacterial presence in the tested plasma samples. We also identified other phages that infect Bacillus bacteria such as the Bacillus virus $\mathrm{G}$ and giant viruses including the Orpheovirus. In three pools (7, 8 and 10), reads belonging to Invertebrate Iridescent Virus 6 with 2966, 145 and 214 reads, respectively were also identified (Figure 1). The additional BLAST search demonstrated that these reads belong to the 392R gene of the iridescent virus genome.

In this study, that analyzed the causes of PDIR among blood donors, primarily commensal viruses with many types of TTV were revealed, which are normal findings. The representatives of the Anelloviridae family are largely disseminated in the human population and can reach very high prevalences of more than $50 \%{ }^{4,5}$. The commensal viruses have not been related to any clinical symptomatology and it is difficult to infer that they can be involved in PDIR. We were unable to identify arboviral agents, however, all of the samples previously positive for DENV and ZIKV by RT-PCR were not included in this study. We were unable to metagenomically identify any known or emerging pathogenic virus in the samples. On one hand, it is possible that some of the PDIR have bacterial etiologies and our bioinformatics pipeline was directed only to viral identification. On the other hand, the majority of the PDIR samples belonged to donors who reported diarrhea, followed by non-specific fever and respiratory symptoms. It is highly likely that, as we tested plasma samples, the viremias of these viruses were very low or did no longer exist and higher viral concentrations could be found in the gastrointestinal and respiratory tracts.

Of note, we were able to identify the presence of many sequence reads belonging to iridescent viruses that infect insects naturally, including mosquito vectors in the plasma samples. We cannot exclude contamination of these sequences during the use of NGS reagents in our experiments, as discussed by other studies ${ }^{6}$. However, we did not use any laboratory reagent that might contain genetic elements or viral particles of insect origin. Another possible explanation for the presence of mosquito sequences is that during the outbreak, the mosquito population was highly abundant in numbers, thus allowing passive transmission of insect viruses to humans. Moreover, some human iridescent virus infections have been described in humans ${ }^{7}$ and it has also been demonstrated that invertebrate iridescent virus 6 can induce interferon-dependent antiviral immune response in mammalian cells ${ }^{8}$. However, more specific and in-depth studies are needed to investigate the possible interactions between mammalian and invertebrate host-specific viruses if these interactions really exist.

In conclusion, the performed viral metagenomic study did not find any pathogenic agent in plasma samples of PDIR cases obtained during a large arboviral outbreak in the Northeast region of Sao Paulo State. This may be a result of the type of symptom reported by the PDIR cases. Nevertheless, viral metagenomics remains a suitable approach for the evaluation of the virome in blood donors and recipients in order to identify emerging viruses threatening the transfusion safety.

$$
\begin{array}{r}
\text { Rafael dos Santos Bezerra }{ }^{1} \\
\text { Kamila Chagas Peronni }{ }^{2}, \\
\text { Bruna Durães de Figueiredo Barros } \\
{ }^{3} \\
\text { Leonardo Scalon de Oliveira } \\
\text { Evandra Strazza Rodrigues }^{1} \\
\text { Rochele Azevedo }^{1} \\
\text { Eugênia Maria Amorim Ubiali }{ }^{1} \\
\text { Dimas Tadeu Covas } \\
{ }^{1} \\
\text { Simone Kashima }^{1} \\
\text { Svetoslav Nanev Slavov }^{\circledR}{ }^{1}
\end{array}
$$

\section{FUNDING}

Fundação de Amparo à Pesquisa do Estado de São Paulo (FAPESP), project $\mathrm{N}^{\circ}$ 17/23205-8, 18/15826-5, 19/08528-0, and 19/07861-8.

\section{REFERENCES}

1. Bezerra RS, Oliveira LS, Moretto EL, Ubiali EM, Silveira RM, Silva Júnior WA, et al. Viral metagenomics in blood donations with post-donation illness reports from Brazil. Blood Transfus. 2020 In Press.

2. Bezerra RS, Jorge DM, Castro IA, Moretto EL, Oliveira LS, Ubiali EM, et al. Detection of influenza A(H3N2) virus RNA in donated blood. Emerg Infect Dis. 2020;26:1621-3.

3. Sauvage V, Gomez J, Boizeau L, Laperche S. The potential of viral metagenomics in blood transfusion safety. Transfus Clin Biol. 2017;24:218-22.

4. Al-Qahtani AA, Alabsi ES, AbuOdeh R, Thalib L, El Zowalaty ME, Nasrallah GK. Prevalence of anelloviruses (TTV, TTMDV, and TTMV) in healthy blood donors and in patients infected with HBV or HCV in Qatar. Virol J. 2017;14:143.

5. Focosi D, Spezia PG, Macera L, Salvadori S, Navarro D, Lanza $\mathrm{M}$, et al. Assessment of prevalence and load of torquetenovirus viraemia in a large cohort of healthy blood donors. Clin Microbiol Infect. 2020 In Press. 
6. Asplund M, Kjartansdóttir KR, Mollerup S, Vinner L, Fridholm $\mathrm{H}$, Herrera JA, et al. Contaminating viral sequences in highthroughput sequencing viromics: a linkage study of 700 sequencing libraries. Clin Microbiol Infect. 2019;25:1277-85.

7. Williams T. Natural invertebrate hosts of iridoviruses (Iridoviridae). Neotrop ntomol. 2008;37:615-32.
8. Ahlers LR, Bastos RG, Hiroyasu A, Goodman AG. Invertebrate iridescent virus 6, a DNA virus, stimulates a mammalian innate immune response through RIG-I-like receptors. PLoS One. 2016;11:e0166088. 